\title{
Motion of a large object in a bubbling fluidized bed with a rotating distributor
}

\author{
A. Soria-Verdugo ${ }^{a}$, N. García-Hernando ${ }^{a}$, J.A. Almendros-Ibáñez ${ }^{b, c}$, U. Ruiz-Rivas $^{a, *}$ \\ a Department of Thermal and Fluids Engineering, Carlos III University of Madrid, Avda Universidad 20, 28911Leganés Madrid, Spain \\ ${ }^{\mathrm{b}}$ Department of Applied Mechanics and Projects Engineering, Castilla La Mancha University, Campus Universitario s/n, 02071 Albacete, Spain \\ ${ }^{\mathrm{c}}$ Renewable Energy Research Institute, C/de la Investigación s/n, 02071 Albacete, Spain
}

\section{A R T I C L E I N F O}

\section{Article history:}

Received 19 November 2010

Received in revised form 14 March 2011

Accepted 7 May 2011

Available online 13 May 2011

\section{Keywords:}

Gas fluidization

Bubbling bed

Distributor

Dead-zone

Object motion

Circulation time

\begin{abstract}
A B S T R A C T
This work studies the effect of a low-frequency rotating distributor on the motion of a large object immersed in a bubbling fluidized bed. The object size and density differ from those of the inert solids that conform the bed. Examples of objects moving in a bubbling fluidized bed include passive particles, catalysts and reactants. The rotation modifies the bed dynamics in the surroundings of the distributor and affects the motion of the object within the bed.

A set of experiments was carried out in a lab-scale cylindrical bed, equipped with a perforated plate distributor that can rotate at around $1 \mathrm{~Hz}$, for different bed aspect ratios, gas velocities, and object characteristics. Sizes were far larger than that of the solids of the dense phase and densities ranged from half the bed density to values around it. The experiments were video recorded, capturing the surface of the bed from above.

As have often been noted, objects might remain in stagnant regions near the distributor and be "lost" or precluded to circulate. This can be avoided in most practical cases forcing the distributor to rotate. Also, the effect of rotation on the circulation time of the objects is presented, showing a general reduction of large circulation times.
\end{abstract}

(c) 2011 Elsevier B.V. All rights reserved.

\section{Introduction}

Bubbling Fluidized Beds (BFB) are used for a variety of applications and in a wide range of scales, from small chemical reactors to large coal combustors. Their main advantages are a good mixing and a large surface area available for chemical reaction or heat exchange. Examples of applications include drying processes, thermal conversion of solid fuels, and coating of particles. In most cases, the presence of large objects within a bed of fine particles is required or happens during the processing, such objects being fuel particles, catalysts, reactants, or agglomerates. Object motion patterns and their ability to move throughout the bed may thus prove to be a key factor in the performance of the bed.

Different measurement techniques have been used both in 2-D and 3-D beds to analyze the motion of objects immersed in the bed. In some of these studies [1-6] the object had a similar density and size to the bed material. These works were intended to characterize the motion of the dense phase. Kunii and Levenspiel [7] reviewed the first studies focused on the motion of large objects in a bed. Rios et al. [8] studied the motion of large objects in 2-D and 3-D beds and discussed the sinking and rising processes. Concerning the rising process, Rios et al. [8] found that the rising motion of objects was

\footnotetext{
* Corresponding author. Tel.: +34 916248463.

E-mail address: ulpiano@ing.uc3m.es (U. Ruiz-Rivas).
}

not a monotonic ascending motion, but the object was lifted small distances by a succession of passing bubbles, rising in a series of small jumps. The relation between the rising velocity of the object and the bubble velocity is complex due to the multiple jumps effect observed by Rios et al. [8] and the relative motion between bubble and object, which are far more important than those between object and dense phase in the sinking process. Nevertheless, the object rising velocity is roughly between $10 \%$ and $30 \%$ of the mean bubble velocity along the bed, according to the experimental works of Nienow et al. [9], Lim and Agarwal [10], and Rees et al. [11]. Several authors have studied the sinking process. Lim and Agarwal [10] found that the sinking velocity of a large object in a 2-D bed was in good agreement with the Kunii and Levenspiel [7] correlation for the velocity of the dense phase for density ratios around 1 . The net buoyant force on an object immersed in a fluidized bed might differ from what would be expected based on its density due to a de-fluidized hood of bed material that appears on top of the object, as stated by Nguyen and Grace [12] and Rees et al. [11]. For large density ratios, Tanimoto et al. [13] and Hoffmann et al. [14] studied the relative motion of sinking objects and dense phase and obtained a linear relation with the density and a potential relation (exponent $1 / 3$ ) with the diameter, but their results cannot be directly extrapolated to the present study since they focused on the motion of binary mixtures, rather than on the motion of a single large object.

In a previous work [15], the motion of a large object in a 2-D bed was studied, and the cycles described by the object, sinking from the 


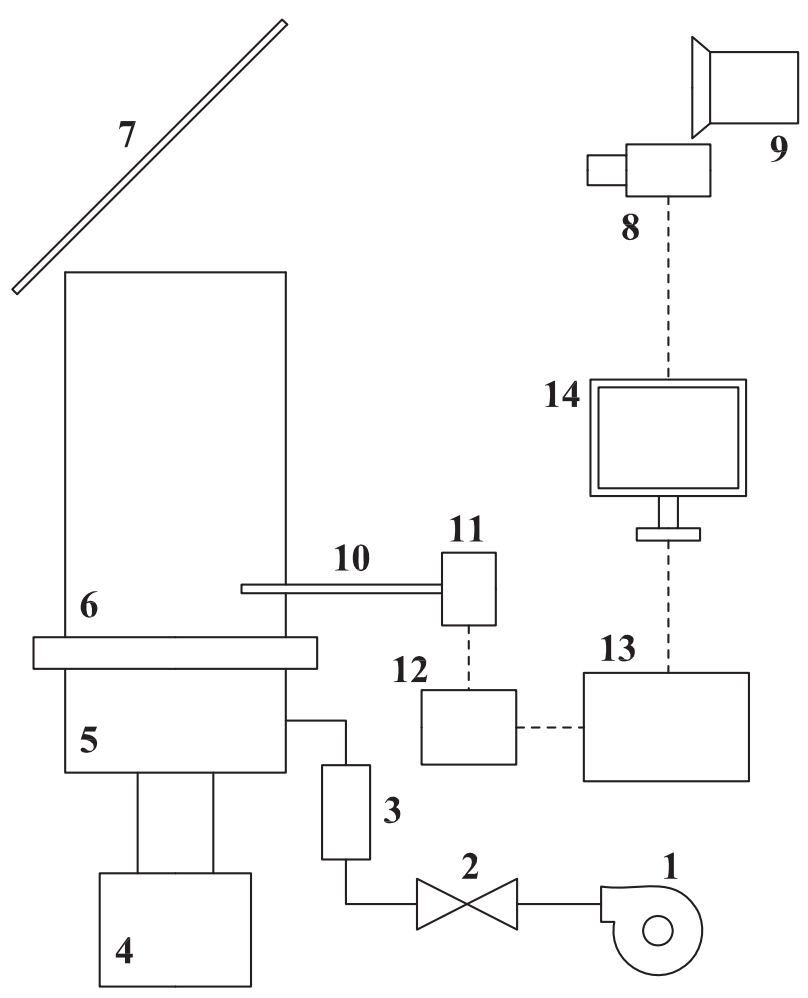

1. Air compresor

2. Regulator

3. Rotameter

4. Electric motor

5. Plenum chamber

6. Bed

7. Mirror

8. Video camera

9. Ligth focus

10. Pressure probe

11. Pressure transducer

12. Pressure signal amplifier

13. Data adquisition system

14. PC

Fig. 1. Schematic diagram of the experimental set-up.

surface of the bed and rising back to it, were characterized. Sinking and rising velocities of the object and its circulation time, which is the time spent in a cycle, were measured. The circulation time was found to depend on the sinking and rising velocities and on two other main parameters: the number of jumps per cycle and the maximum depth attained. The relative frequency of cycles with a certain number of jumps decreases exponentially with the number of jumps, a result that suggests that the ability of a bubble to raise an object to the surface is roughly constant, independent of the bubble size. On the other hand, the relative frequency to attain a certain depth was parabolic, with a minimum at medium depths. This is due to the fact that the object usually sinks in the sides of the bed and at medium depths bubbles seldom appear in such zones.

Finally, most of the authors $[8,9,12,15,16]$ noticed that the object, quite regardless of its density, may stop circulating within the bed and be captured in stagnant zones over the distributor. Sanderson and Rhodes [15] showed that the reappearance of a stagnant object was possible increasing the gas velocity over $3 U_{m f}$. Also, by analyzing experimental data for various 3-D bed sizes, they showed that the motion of objects inside the bed was a scalable phenomenon, following hydrodynamic similarity criteria for bubbling beds.

In this paper, a comparison of the results obtained in a standard bubbling fluidized bed (BFB) and in a "forced" BFB is presented. Several works have proposed the use of different actuators, with the aim of modifying the dynamics of the BFB. Passive or active control tools are motivated by general drawbacks of BFB, such as poor radial mixing, large bubbles and gas by-pass, or agglomerates and the incidence of heterogeneous fluidization. Proposed solutions include pulsed gas flow ([17,18]), vibrating beds [19,20], and the use of advanced distributor designs, including spiral [21,22], swirl [23] and rotating distributors [24,25].

Sobrino et al. [24], using a similar experimental setup to the one used in the present experiments, showed that a rotating distributor may affect global bed parameters. For instance, they showed that, as a consequence of rotation, bubble diameter is affected, and become more homogeneous along the bed and minimum fluidization velocity decreases.

This work studies the effect on the bed dynamics of allowing the bed distributor to rotate, by means of studying its effect on the motion of an object submerged in the bed. The experimental tests were performed on a lab-scale cylindrical BFB, equipped with a perforated plate distributor that can either remain static or rotate, at a low frequency around $1 \mathrm{~Hz}$. Also, the influence of gas velocity, bed height, and the density and shape of the object was analyzed throughout the experiments for both the static and the rotating distributor configurations.

\section{Experimental setup}

The experimental setup consists of a lab-scale cylindrical BFB, an electrical motor that allows the distributor to rotate and an image acquisition system to capture the freeboard. The bed performance was characterised by means of pressure measurements. Fig. 1 shows a schematic diagram of the experimental setup with the main components.

The cylindrical vessel for the bed is a transparent tube with an inner diameter $(D)$ of $0.192 \mathrm{~m}$ and $1 \mathrm{~m}$ in height. The fixed bed height $\left(h_{b}\right)$ was varied throughout the experiments between $0.25 D$ and $0.75 D$. The minimum fluidization velocity was measured to be $0.31 \mathrm{~m} / \mathrm{s}$ for the rotating distributor bed and $0.33 \mathrm{~m} / \mathrm{s}$ for the static one, thus the minimum fluidization velocity slightly decreases with the rotation, in agreement with Sobrino et al. [24].

Sand with a measured density of $2,632.5 \mathrm{~kg} / \mathrm{m}^{3}$ and a particle size distribution as shown in Fig. 2 was used as bed material. According to Geldart's classification [36], these particles are type B. The bulk density of the fixed bed was measured to be $1630 \mathrm{~kg} / \mathrm{m}^{3}$, which gave the void fraction of the packed bed, $\varepsilon_{\text {packed }}=0.38$. The diameter of the cylindrical object $\left(D_{O}\right)$ was $6.4 \mathrm{~mm}$ while its den- 
Table 1

Density $\left(\rho_{0}\right)$ and length $\left(L_{0}\right)$ of the cylindrical objects used in the experiments. Diameter $\left(D_{O}\right)$ was always $6.4 \mathrm{~mm}$.

\begin{tabular}{lll}
\hline & Density, $\rho_{o}\left(\mathrm{~kg} / \mathrm{m}^{3}\right)$ & Length, $L_{O}(\mathrm{~mm})$ \\
\hline$a$ & 1380 & 6.4 \\
$b$ & 1380 & 19.2 \\
$c$ & 1380 & 32.0 \\
$d$ & 840 & 19.2 \\
$e$ & 1010 & 19.2 \\
$f$ & 1750 & 19.2 \\
\hline
\end{tabular}

sity and length was varied throughout the experiments, as shown in Table 1 . These values are typical for a biomass pellet.

Therefore, objects $a$ to $e$ are flotsam particles (buoyant) within the fluidized bed, while object $f$ is a jetsam particle.

The distributor is a $6 \mathrm{~mm}$ width perforated plate with 275 holes of $2 \mathrm{~mm}$ diameter, distributed in a triangular mess of $11 \mathrm{~mm}$ pitch. According to Karri and Werther [26] and Kunii and Levenspiel [7] the pressure drop along the distributor should be larger than $30 \%$ of the pressure drop along the bed for an homogeneous fluidization; in the present work, the pressure drop along the distributor $\left(\Delta P_{\text {dist }}=5200\left[\mathrm{~Pa} /(\mathrm{m} / \mathrm{s})^{2}\right] \mathrm{U}^{2}\right)$ is $30 \%$ of the pressure drop along the bed for an air velocity of 1.25 times the minimum fluidization velocity and a bed aspect ratio of 0.75 . Therefore throughout the experiments (velocities larger than $1.25 U_{m f}$ and/or bed aspect ratios smaller than 0.75 ) the condition is always fulfilled. Moreover, the pitch was selected to minimize the existence of dead zones near the distributor, following the study of Geldart and Baeyens [27]. More recently, Rees et al. [28] presented a new criteria related to the jet diameter, which they calculate using magnetic resonance. Following their study and correlations, in the present work the pitch that minimizes the dead zones should be of around $8 \mathrm{~mm}$ (with a small variation for the different experimental conditions), a result in fair agreement with our selection, which follows the standard procedure. The distributor might be forced to rotate at a maximum constant velocity of $100 \mathrm{rpm}$ by an electrical motor.

Pressure fluctuations inside the bed were measured through a $4 \mathrm{~mm}$ internal diameter and $50 \mathrm{~mm}$ long steel tube connected to a piezo-electric pressure transducer (Kistler type 5015). The signal from the piezo-electric sensor was amplified by a Kistler amplifier. The response of this kind of probe and transducer is in good agreement with the model of Bergh and Tijdeman [29], as stated by van Ommen et al. [30]. This model predicts the first resonance frequency to occur at $670 \mathrm{~Hz}$, which is far away from typical characteristic frequencies in fluidized beds (in the $1-5 \mathrm{~Hz}$ range). The sampling frequency was $200 \mathrm{~Hz}$ and 12,032 samples per test were recorded. The amplifier acts as a high-pass filter with a frequency of $0.16 \mathrm{~Hz}$, in order to obtain the pressure fluctuations relative to

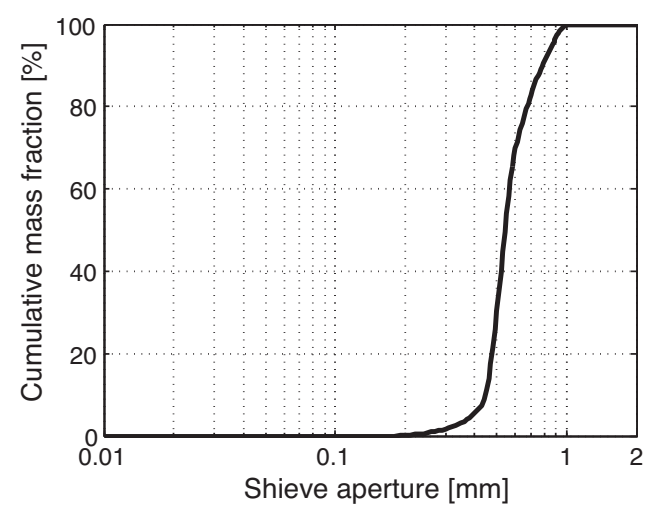

Fig. 2. Particle size distribution of the bed material.
Table 2

Experimental parameters. Nominal case is in bold format.

\begin{tabular}{ll}
\hline Bed aspect ratio $H / D$ & $(0.25 ; \mathbf{0 . 5} ; 0.75)$ \\
Excess air ratio $U / U_{m f}$ & $(1.26 ; \mathbf{1 . 4 4} ; 1.63)$ \\
Object aspect ratio $L_{O} / D_{O}$ & $(1 ; 3 ; 5)$ \\
Bed-object density ratio $\left(\rho_{b}-\rho_{o}\right) / \rho_{b}$ & $(-0.07 ; \mathbf{0 . 1 5} ; 0.38 ; 0.48)$ \\
Rotating angular velocity $n(\mathrm{rpm})$ & $(0 ; 100)$ \\
\hline
\end{tabular}

the local average pressure. The signal was also low-pass filtered at the Nyquist frequency by the amplifier.

The characteristic frequency of the fluidized bed was determined by a frequency domain analysis for the nominal case. Fig. 3 shows the power spectra using the Welch [31] method, with all sub-spectra based on 4096 samples. Considering the sampling frequency, these power spectra have a frequency resolution of $0.049 \mathrm{~Hz}$, which is a common and accepted value [32].

The power spectra show that the fluidized bed characteristic frequency $(3.61 \mathrm{~Hz})$ is not affected by the rotating distributor, but the signal energy is almost doubled when the distributor rotates.

During all the tests, the fluidized bed freeboard was video recorded during $10 \mathrm{~min}$ with a recording speed of 30 frames per second. The resolution of the camera was $480 \times 640$ pixels. The videos were post-processed using Matlab ${ }^{\circledR}$. Both the object positions while on the bed surface and the time periods of disappearance from it (the circulation time of the sinking-rising global cycle) were obtained from the videos. The experimental results are based on number of cycles and circulation time measurements. The object radial and azimuthal motion in the freeboard was also studied, giving little relevant results. Concerning the radial motion, it was observed that the object was more prone to appear at the centre of the bed (by the action of bubbles) and sank at the sides (following the dense phase), in accordance with previous works on bubble or object paths [33-35]. Concerning the azimuthal motion, no relevant pattern was observed throughout the experiments.

Experiments were run for a range of gas velocities, bed heights and object characteristics and for both the static and the rotating distributor configurations of the BFB. Test parameters are shown in Table 2, where $h_{b}$ is the total bed height and $D$ is the bed diameter, $U$ and $U_{m f}$ are the air velocity and the minimum fluidization velocity, $L_{O}$ and $D_{O}$ are the object length and diameter $\rho_{b}$ and $\rho_{o}$ are the (packed) bed density and the object density and $n$ is the rotating angular velocity. The nominal case selected for comparison is highlighted in bold type in Table 2 . Whereas one parameter was varied in the experiments, the rest of them were kept as in the nominal case.

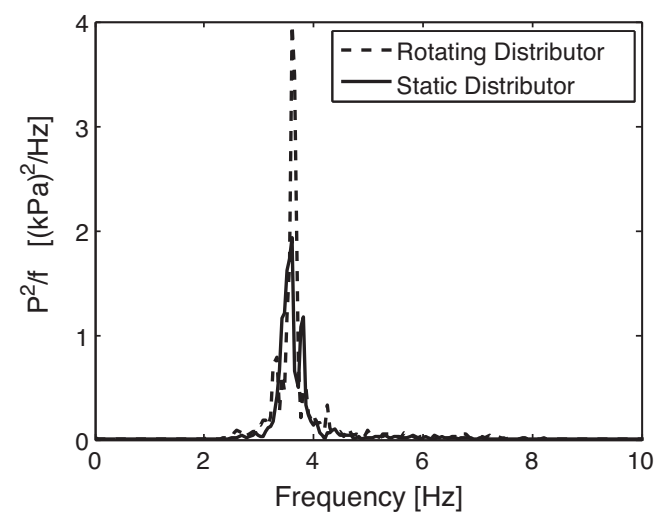

Fig. 3. Power spectra for the rotating and static distributor configurations. Nominal case $\left(U / U_{m f}=1.44, h_{b} / D=0.5\right)$. 


\section{Results}

The experimental results are grouped in two sections. In the first one, the process of object stagnation is studied. In the second, a general study of the circulation time distribution is presented.

\subsection{Object stagnation}

The first key point observed was that the object did not appear at the freeboard after a determined time when operating with the static distributor. Every time the object disappeared, the bed material was extracted in order to find its position. It was always found to be settled in a stagnant position at the bottom of the bed, placed on the small dead zones between the holes of the distributor (a triangular mess of $11 \mathrm{~mm}$ pitch, with object diameter $6.4 \mathrm{~mm}$ and lengths ranging from 6.4 to $32 \mathrm{~mm}$ ). As stated in Section 1 , this behaviour has been often reported in the literature as a typical drawback of non-porous (non-homogeneous) distributors. Although special care was taken in the design of the distributor, object stagnations happened for almost all the tests (with the static distributor). Only for very light objects and large bed aspect ratios the stagnant probability of the object could be considered negligible.

Nevertheless, when operating with the rotating distributor, those object stagnations were completely inexistent. Moreover, if the distributor was forced to rotate after the object was stagnated in a static distributor configuration, it reappeared almost immediately, in a 3-5 s lapse. This time lapse should not be considered as the time needed for the object to arrive to the top, because also a certain starting time is needed for the electrical motor to acquire the operating frequency. The fact that the rotating distributor is even capable of maintaining in circulation an object denser than the bed (object $f$ ) is significant. Far denser objects (around the dense phase density) did not circulate, independently of the distributor, and were immediately stagnated. If the incidence of object stagnations should be avoided, the distributor rotation may prove a better methodology to "activate" the circulation of stagnant objects with intermediate densities than increasing the gas velocity, as suggested by Sanderson and Rhodes [15], since the loss of fines will be avoided. Obviously, the feasibility of a rotating distributor will strongly depend on the bed geometry and dimensions and it will be generally restricted to small scale applications.

Object stagnation is of course a bottom zone mechanism. That zone is characterized by the distributor geometry, and will show both air jets and de-fluidized zones (minimized by the pitch selection), as shown by Geldart and Baeyens [27] and Rees et al. [28]. The rotation of the distributor: (i) varies the jet location, and (ii) is able to move the bottom bed material, producing a $3 \mathrm{~cm}$ shear layer (rough estimation, observed by visual inspection) in the defluidized particles. These two superposed effects completely avoid the formation of stagnant regions and are able to immediately restore the object into circulation.

This effect of preventing object stagnations is the main result of these experiments. Some results of the incidence of object stagnations in static distributor beds follows. The main interest of these results is to state in which cases the preventing effect of the rotating distributor is more relevant. With such a purpose, ten tests of $10 \mathrm{~min}$ were video recorded and post-processed for each experimental condition in order to determine the stagnation cycle probability and time needed for the object to be stagnant in each configuration with the static distributor. Ten is not a statistically significant number to guarantee the repeatability of the results, but the aim of this analysis is only qualitative. The stagnation cycle probability is presented in Eq. (1). This parameter is a more reliable factor than the time till the object sinks for the last time. It seems reasonable to consider each cycle of the object independent of the previous history, and the time till the object sinks for the last time will of course be influenced by the circulation time of the previous cycles of the object, which distribution will be discussed in the following section.

$P_{S C}=\frac{1}{N_{a p}+1}$

The stagnation cycle probability defined in Eq. (1) represents the probability for an object to be precluded to circulate. This means that an object which is settled over the distributor after appearing at the freeboard twice, would define a stagnation cycle probability of 0.33 , meaning that the object experienced three sinking paths and two rising paths or, in other words, that a particle following this behaviour will have, when starting to sink, a $67 \%$ probability of returning to the surface and a $33 \%$ probability of reaching a stagnant position and remaining in it for a large period of time, being precluded to circulate.

Results for the stagnation cycle probability in a static distributor configuration are shown in Fig. 4. The mean value and the maximum and minimum values ( \pm interval) of the stagnation cycle probability are plotted as a function of the experiment varying parameters. The nominal case (highlighted) is plotted in all the graphs for comparison. According to the definition of stagnation cycle probability, the results have been presented with a log scale in the probability axis. The four graphs of Fig. 4 present the separated effects of bed aspect ratio and gas velocity, object density and object aspect ratio on the stagnation cycle probability. It is not straightforward to compare these results with those observed using the rotating distributor configuration, as in those cases no stagnations were observed (and the probability will then be 0 ). Nevertheless, the experiments were performed during a determined period of time, sufficiently large to ensure that at least 100 cycles were completed for each rotating distributor case. Therefore, the stagnation cycle probability for any static distributor configuration can be said to be at least smaller than 0.01 and this can serve, in comparison with the data in Fig. 4, to elucidate the effect of the rotating distribution.

Fig. 4(a) and (b) presents the influence of the BFB dynamics, and Fig. 4(c) and (d) that of the object characteristics. As explained above, the results can only be considered as qualitative information, so minor changes will not be discussed. With such a consideration, three different behaviours can be disregarded. First, those configurations in which the object rapidly stopped circulating, corresponding to values of the stagnant cycle probability between 1 and 0.1 , meaning according to Eq. (1) that the object appeared between 0 and 9 times at the bed surface before stagnation. Such cases include the nominal case and those for a shallow bed, a denser object or a larger one. Here the effect of the rotating distributor is extremely remarkable, since there is a high probability for the object to settle over the distributor when operating with the static distributor while the object never reached the stagnant zones when operating with the rotating distributor. Next, the configurations in which the object stopped circulating after a rather large number of cycles can be consider. They show values of the stagnation cycle probability between 0.1 and 0.01 , meaning $10-100$ object cycles before being stagnant. Such cases correspond to smaller or larger gas velocities, a lighter object or a smaller one. Here the effect of the rotating distributor is still important.

Last, there are those configurations in which the object did not stop circulating during the $10 \mathrm{~min}$ test for both static and rotating distributor configurations. In such cases, as explained previously for the rotating cases, the experimental value of the stagnation cycle probability is zero or, more precisely, it is at least smaller than one over the number of cycles that the object has performed during those $10 \mathrm{~min}$. Those cases are for a deep bed $\left(h_{b} / D=0.75\right)$ and for a low density object $\left(840 \mathrm{~kg} / \mathrm{m}^{3}\right.$, which gives a bed-object density 

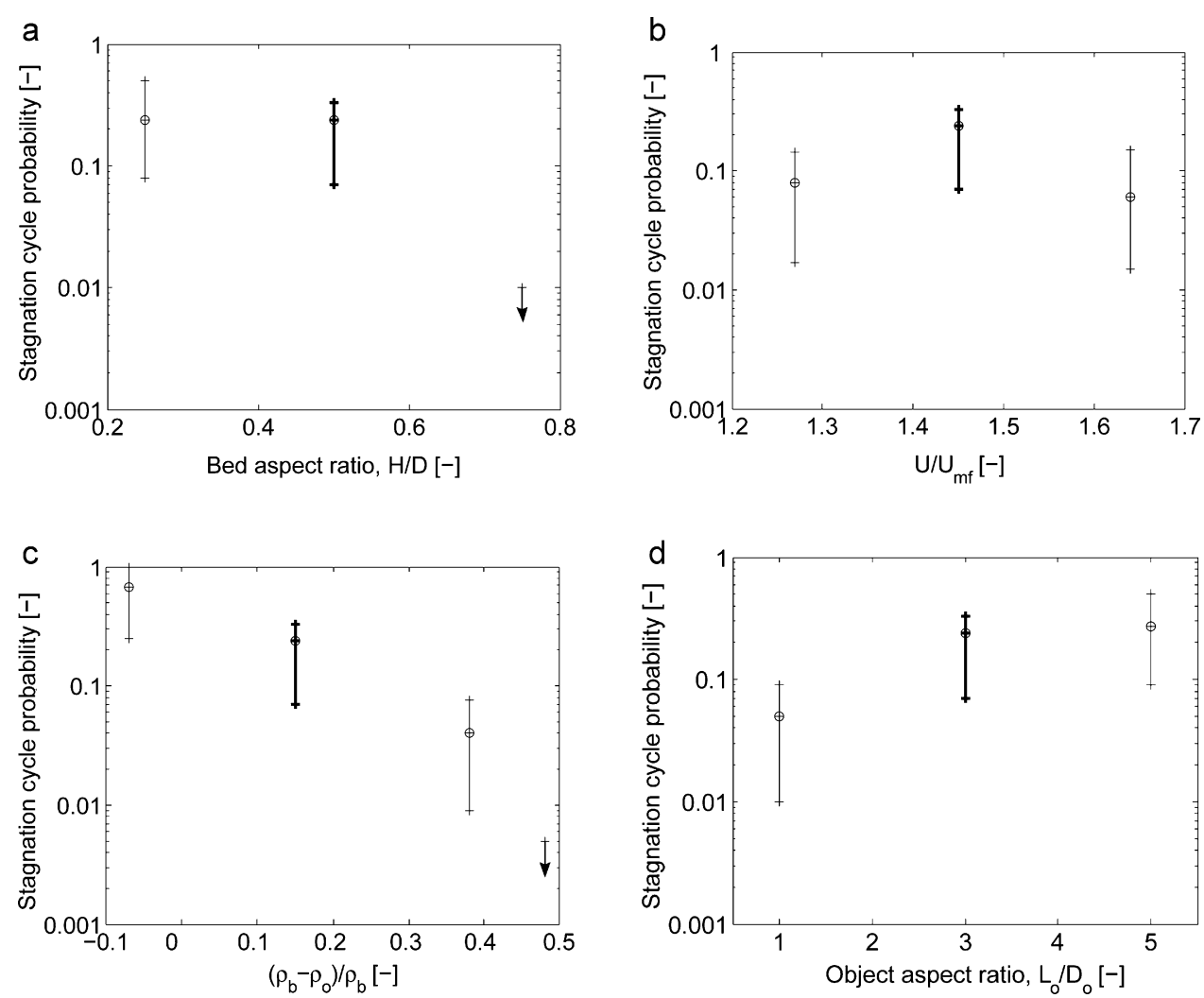

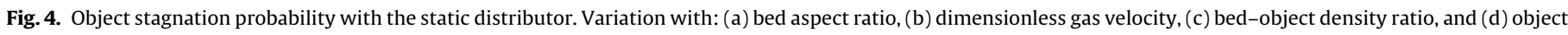
aspect ratio.

ratio of 0.48 ). In the graphs they are represented by a dot and an arrow, meaning that their probability is at least smaller than the given value. Here the rotation of the distributor is of no use as far as the stagnant cycle probability is concerned. In both cases, it is clear that the ability of the object to reach the bottom zone is reduced. First by a larger depth and thus a need of a larger residence time in the dense phase, and also because of the existence of larger bubbles near the freeboard, with increasing probability to interact with the object. And second by a lower density, and thus buoyant forces becoming more important and precluding the object to sink deep. This last case is not really relevant, as the object merely stayed in the bed surface and did not circulate homogeneously throughout the bed. On the contrary, the restriction for shallow beds is important, and the effect of the distributor rotation is slight for deep beds, where object stagnations seldom appear.

The effect of bed aspect ratio and object density on the stagnation cycle probability seems quite straightforward. Above a certain height, the probability for an object to reach the bottom zone rapidly diminishes for the reasons suggested in the previous paragraph. Decreasing the object density and thus increasing the related buoyant forces also decrease the probability of the object travelling to the bottom. Therefore, in both cases, losses are prevented. The influence of air velocity and object aspect ratio on the stagnation cycle probability was not so straightforward. The results in Fig. 4 (b) suggest the existence of competing mechanisms when the gas velocity changes. Decreasing stagnant probabilities for increasing gas velocity was the expected result, as the intensity of the fluidization is improved [37], and both the rising and sinking velocities are increased. The low probability datum for the lower gas velocity might be related to a change in the bed structure, that is, the gas velocity might be too low for establishing the typical 2-vortex configuration, as shown by Pallarès and Johnsson [35], with a preferential path for bubbles at the middle of the bed. Finally, Fig. 4(d) shows that the incidence of object stagnations decreases for smaller objects, while no difference is observed between the two larger objects (aspect ratios 3 and 5). It seems reasonable that objects with larger aspect ratios (which means larger objects, as the diameter of the cylindrical objects is constant) could not be easily raised by bubbles due to their larger inertia. In contrast, smaller objects could be easily raised. Nevertheless, this is in fact beyond the purpose of this work, which is focused in establishing the effects of the rotating distributor.

Industrial applications often rely on the very good vertical mixing associated with fluidized beds, so that the ability of large objects to move through all zones within the bed (including the bottom zone) is envisaged. Therefore, object stagnation may occur and might cause minor or severe problems, depending on the application, including reactant loss, hot spots, agglomeration, corrosion, etc. A method of re-releasing the objects is therefore necessary, and an actuator applied to the distributor, such as a low frequency rotation, may be a more efficient tool than increasing the gas velocity for small scale applications. The rotation of the distributor can be used in applications such as coating reactors, where the efficiency depends on the appearance of all the particles to be coated at the freeboard, small dryers where the motion of aggregates should be enhanced to improve the fluidization quality, or small reactors where a homogenous temperature is intended.

\subsection{Circulation time distribution}

The distribution of circulation times for the object was also considered in this study to characterize the effect of the rotating distributor. The circulation time datasets were obtained performing $10 \mathrm{~min}$ tests for all the experimental configurations. The number of cycles was always beyond 100 , which can be considered an adequate number for statistical analysis. Fig. 5 shows the histograms 

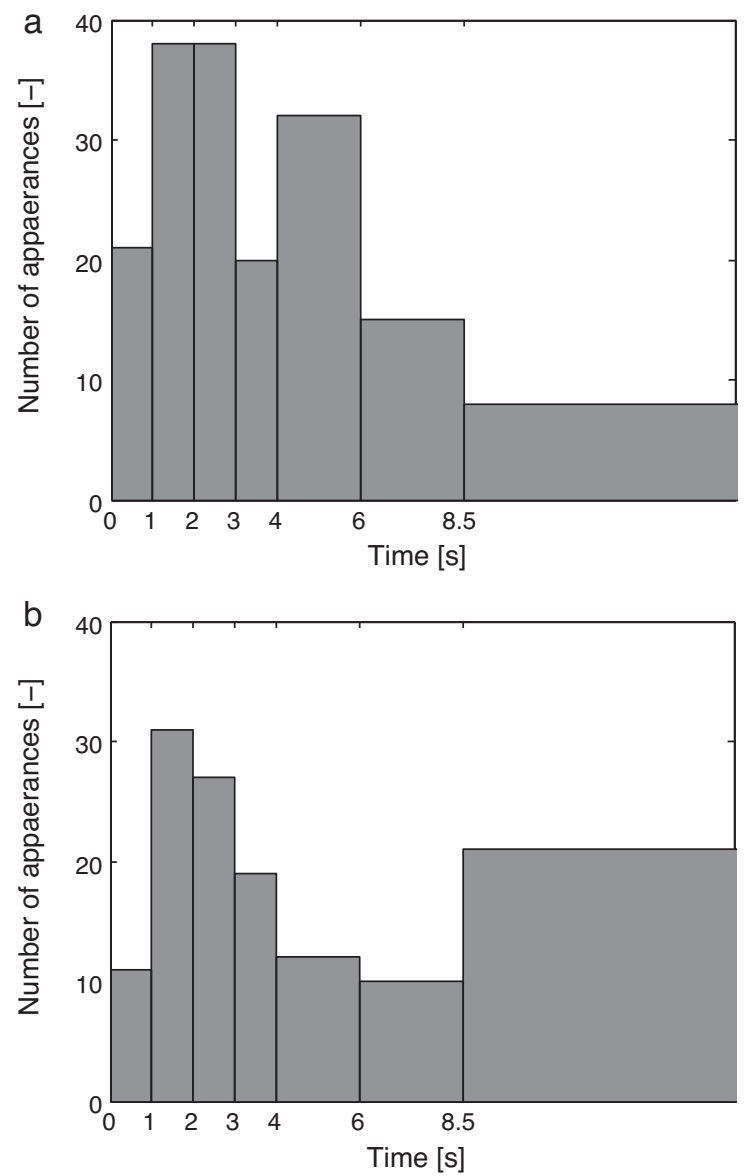

Fig. 5. Histograms of the circulation time for the object to return to the bed surface: (a) rotating distributor, (b) static distributor. Nominal case $\left(U / U_{m f}=1.44, h_{b} / D=0.5\right)$.

of the circulation times for both the rotating and the static distributor configurations. In both cases, the experiments were run for the nominal experimental conditions $\left(U / U_{m f}=1.44, h_{b} / D=0.5\right.$ and nominal object parameters). Time intervals in the histograms were chosen to give a sufficient number of appearances in each bar $(>5)$, for all the experimental conditions. For the static distributor, the object stagnations are taken into account in the $t>8.5 \mathrm{~s}$ interval.

Chi-square tests of population homogeneity were performed, as a first step to establish that different datasets correspond to different dynamics, and thus the effect of the rotation on the circulation times is beyond statistical uncertainty. The Chi-square test of population homogeneity between the histograms of the two datasets gives a $p$-value, and values smaller than 0.05 mean that there is sufficient statistical evidence to guarantee that the two datasets are different. Chi-square tests of population homogeneity were performed between static and rotating distributor measurements maintaining all other conditions identical. The result for the nominal case showed a $p$-value of 0.012 , so the two sets can be considered different, and a certain effect due to the rotation did exist. But, of course, the mechanisms related to object stagnations have already been shown to be extremely different with and without rotation. This mechanism affected the data for large times, although it is not the only cause for large circulation times. Repeating the Chisquare test disregarding the $t>8.5 \mathrm{~s}$ data for both the static and the rotating distributor cases gives a new $p$-value of 0.27 , so there is no statistical evidence to guarantee that these two datasets correspond to different dynamics. Therefore, the effect of the rotating distributor is only evident for cycles of large circulation time. This means that the rotation only affects the bottom of the bed, and not the regions above that zone. Chi-square tests were performed for all the experimental conditions defined in Table 2, giving similar evidence in all the cases.

The circulation time was calculated for all the experimental conditions as the time lapse between consecutive appearances of the object at the bed surface. The medians of all the circulation time distributions are shown in the graphs of Fig. 6 for comparison with the rest of conditions, varying object and bed parameters, and with and without rotation.

There is an absence of data in three experimental conditions corresponding to the static distributor configuration. In those cases the object was stagnated in the different experiments after a small number of cycles, so there was not enough data for a proper estimation. Those cases are for a shallow bed $\left(h_{b} / D=0.25\right)$, for a high density object $\left(1750 \mathrm{~kg} / \mathrm{m}^{3}\right.$, higher than the bed density, which gives a negative bed-object density ratio of -0.07 ), and for an object of high aspect ratio $\left(L_{O}=32 \mathrm{~mm}\right.$, which gives an object aspect ratio of 5). In all these cases, the object seldom appeared back in the freeboard throughout the experiments. This was also the case for the nominal conditions, but in that case a far larger number of experiments were performed in order to obtain a sufficiently large dataset.

The results for the median show just a slight reduction when using the rotating distributor. Therefore, there is no relevant effect of the distributor rotation, apart from the cases where the absence of data for the static distributor shows the ability of the rotating distributor to maintain the object in circulation. This was rather expected, as it has been shown that only large circulation times were modified and the median is a parameter that remains quite unaffected with such variations.

On the other hand, the results in Fig. 6 show the effect of the varying parameters on the circulation time. As has been done previously, a first step is to establish that the different datasets shown in each graph correspond to different dynamics, and thus that the differences are beyond statistical uncertainty. For such a purpose the Chi-square tests were employed. Therefore, tests of population homogeneity were carried out between the datasets for each experimental condition and the nominal cases. Chi-square tests showed $p$-values always smaller than 0.05 for all the combinations. Thus, the influence of the different parameters (bed aspect ratio, dimensionless gas velocity, object density and size, and rotation) on the circulation of the objects can be considered as well established.

The results showed an increase of the median value of the circulation time for increasing bed aspect ratios and a slight increase for increasing gas velocities. The first effect could be attributed to the average depth attained in a cycle, a parameter that will reasonably increase for larger bed depths. The effect of the gas velocity is not so straightforward. It would seem reasonable to expect a reduction of the circulation time for increasing gas velocities. Such an increase will produce higher sinking and rising velocities, according to the well established correlations for the bubble and dense phase velocities [7,34]. No definitive explanation is given, as the effect is not very pronounced and it is less evident for the whole dataset, as will be seen in Fig. 8.

When varying object parameters, the median value decreased for increasing object aspect ratios and bed-object density ratios, although in this last case, the large difference between the flotsam objects and the jetsam object was the main feature. This might be explained by the fact that the jetsam object reached the bottom zone in practically every cycle. Therefore, its dynamics were associated with the rotation and the related mechanisms that released the object from such a zone. This is in accordance with the results presented in the object stagnations section. On the other hand, most of the flotsam objects cycles will be restricted to the upper layer, and the median represents these cycles. The effect of the object aspect 

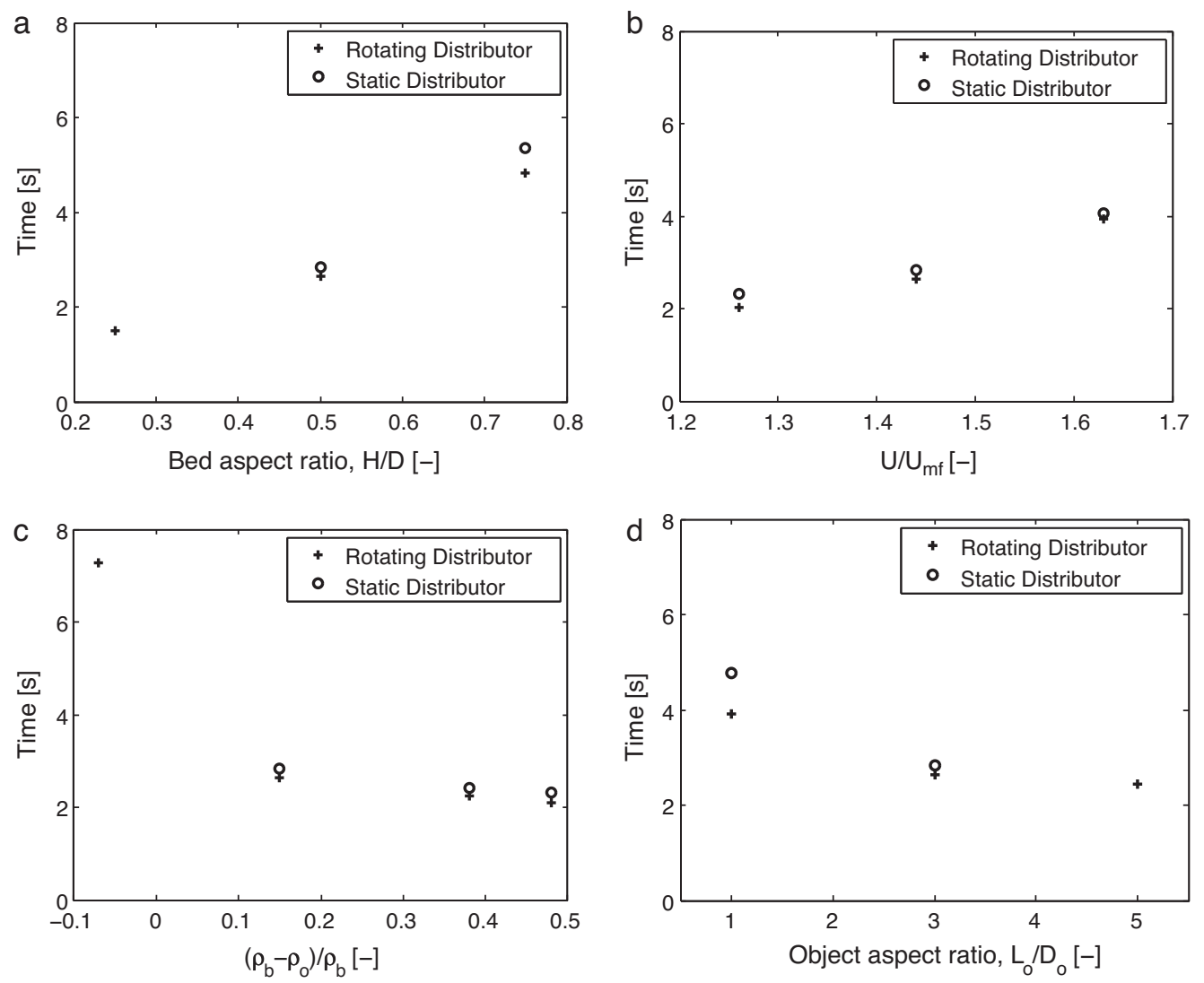

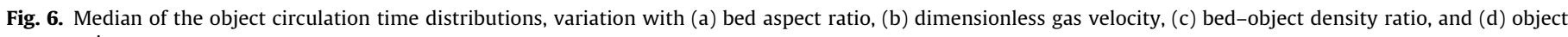
aspect ratio.

ratio might be attributed to the higher buoyant forces suffered by a larger object.

For a deeper analysis of the distributor effect, the information of the median is not sufficient. To give information of the larger circulation times, box plots were used rather than histograms, as they give the information in a more compact form. Box plots are commonly used for a brief understanding of a statistical dataset. In a box plot, the information is reduced to the median, the lower and upper quartiles (representing $25 \%$ and $75 \%$ of the population), a confidence interval (defined by the two experimental data that are further away from the median but inside a maximum interval of \pm 1.5 times the inter-quartile range from the quartiles), and outliers (that lie out of such a confidence interval). Fig. 7 shows the box plots of the data given in Fig. 5. There is a significant difference concern-

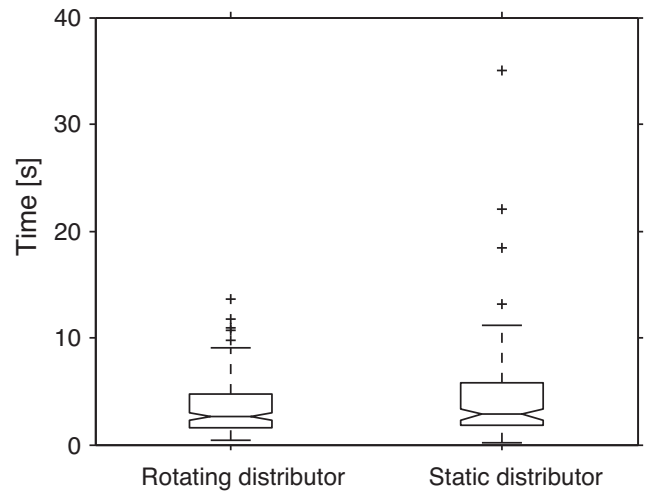

Fig. 7. Box plots of the circulation time distributions in Fig. 5. Nominal case $\left(U / U_{m f}=1.44, h_{b} / D=0.5\right)$. ing outliers. The stagnant object data for the static distributor were included in the calculation of median and intervals, although they were not shown as outliers. Not considering these stagnant objects would lead to miscalculations of the statistical values presented in the boxplot. Note that for the median and intervals calculation the only important fact is that they represent large times, but it is unimportant to know how large (as is the case for the calculation of the mean or the standard deviation). They were not pointed as outliers because the time required to rise to the freeboard is unknown, although at least larger than the experiment runtime.

The eight graphs of Fig. 8 present the box plots of the circulation time datasets as a function of bed aspect ratio and dimensionless gas velocity (note the change of scale for this plot), object density and object shape, considering both the static and the rotating distributor configurations. The nominal case shown in Fig. 7 is highlighted in the eight graphs.

Once again, the effect of maintaining the object in circulation when rotation is applied to the distributor is observed in those graphs where the data for the static configuration is missing. Apart from that, the effect of the distributor in the incidence of large circulation times can be observed in most cases, the distributor rotation being a mechanism to shorten those times. This is probably due to its effect in releasing the object from the quasi-stagnant regions near the distributor plate. Such an effect is evident, and probably enhanced, for varying object size or density.

The results presented in this work show a clear effect of the rotation on the object motion parameters. This can be explained in a mechanistic way. The rotation of the distributor affects the dynamics at the bottom of the bed. The rotation varies the layout of gas jets and moves the bottom bed material, generating a shear layer of about $3 \mathrm{~cm}$ over the distributor. When the object is at the bottom of the bed, the distributor rotation and the centrifugal 
a

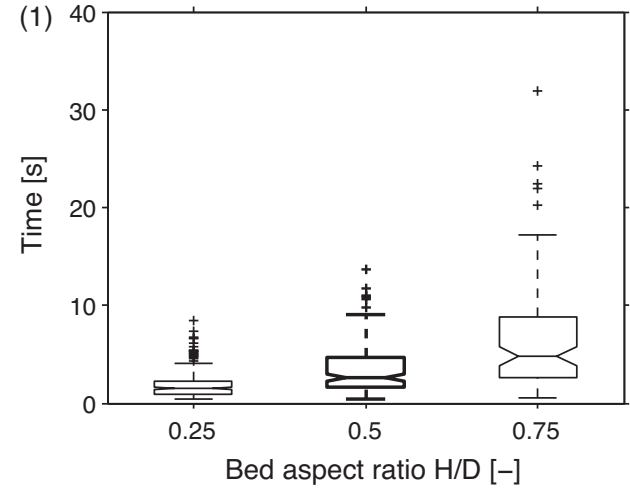

b

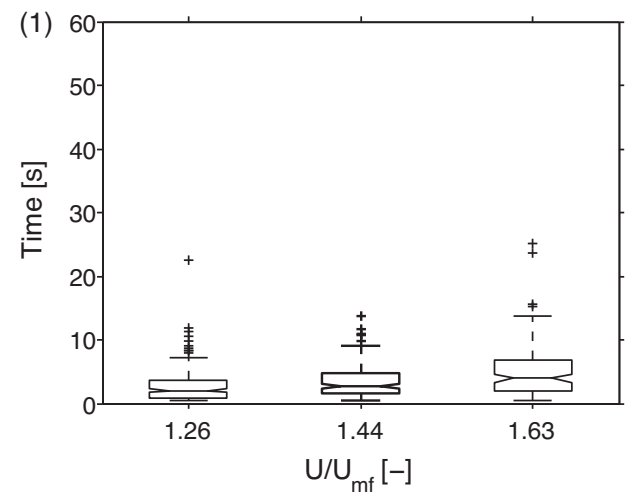

C

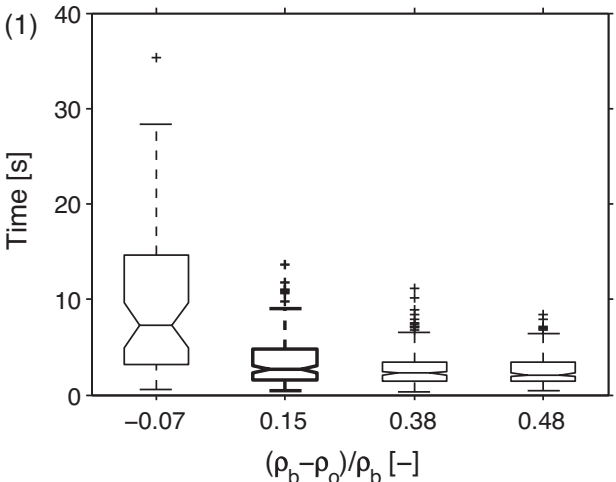

d

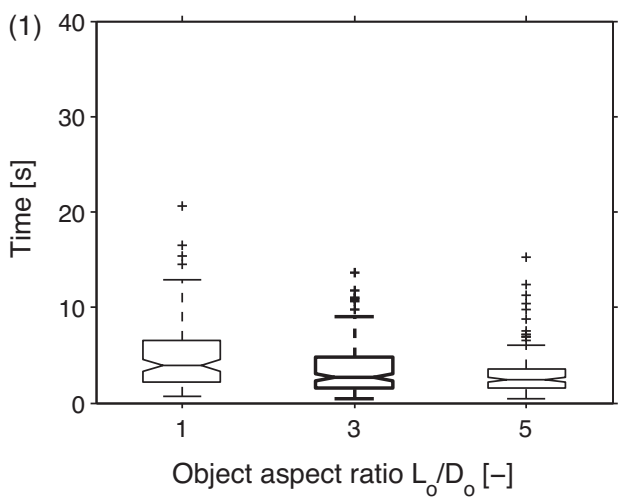

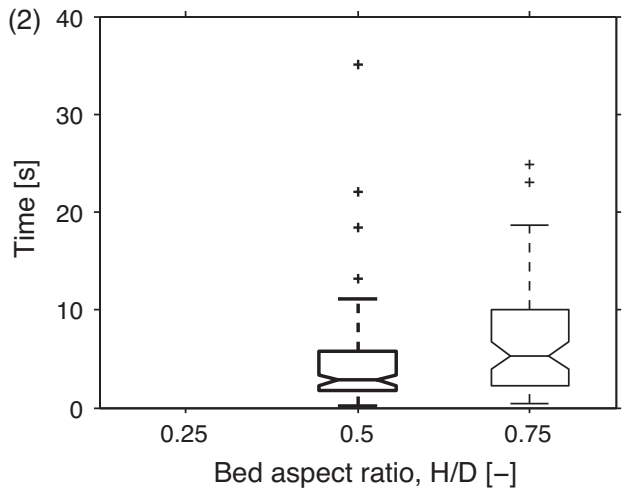
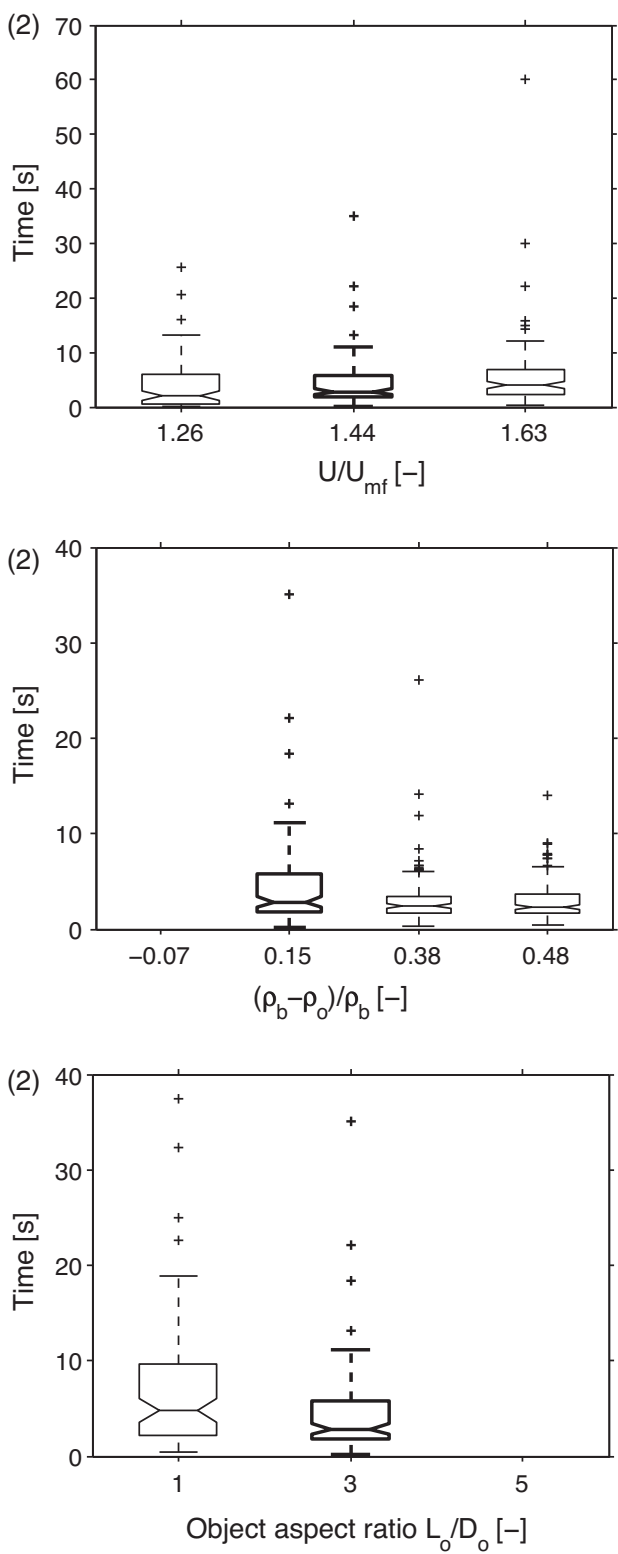

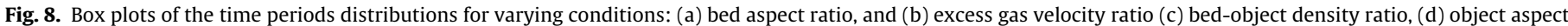
ratio; (1) rotating distributor, (2) static distributor. 
force derived from it produce an azimuthal and radial motion. This motion increases its probability to interact with a jet. The object has thus a higher probability to rise to the bed surface. If the distributor is static, the object does not have this motion, reducing its probability to move out of stagnant regions to the surroundings of a gas jet. Consequently, the circulation times are larger when operating with the static distributor. Of course, the power consumption needed to rotate the distributor and the inherent complications of imposing rotation on a large distributor will generally condition its applicability to small scale.

\section{Conclusions}

The motion of a large object within a lab-scale BFB was analyzed, operating with a rotating distributor or in a static distributor conventional bed. Several tests were carried out for a range of bed characteristics (bed height and gas velocity) and object parameters (density and size).

During the operation with the static distributor, after a number of cycles out and back to the surface of the bed, the object ended motionless in the small dead zones between holes over the distributor, for almost all test configurations. This effect has often been referred in the literature but was not the case for the rotating configuration, where no object stagnations were reported. In some cases, for large and dense objects, or operating in a shallow bed, and even for the nominal conditions of the experiments, the rotation of the distributor permitted the continuous motion within the bed of objects that were immediately stagnated and did not circulate at all with the static configuration. Moreover, when an object was stagnated in a static configuration it could be recovered almost immediately, by forcing the distributor to rotate. The rotation breaks the layout of gas jets and stagnant zones just above the distributor, improving the radial and axial mixing at the bottom of the bed.

The circulation time distribution was presented for the different configurations and varying parameters. A statistical analysis of the data was performed, showing a slight effect of rotation in large circulation times. The distribution shows that most of the results are confined in a small interval (around $0-5 \mathrm{~s}$ for most configurations), and then there is a series of outliers (going up to $40 \mathrm{~s}$ ) that describe the physics of multiple-jump rising and bottom bed phenomena. The rotation was found to strongly affect the dynamics of such cycles.

Therefore, rotation of the distributor (either continuous or applied at given intervals) might prove useful when the circulation of objects throughout the bed is envisaged and object stagnations or large circulation times should be prevented.

\section{Acknowledgments}

This work has been partially supported by the National Energy Program of the Spanish Department of Science and Education (ENE2006-01401), the Spanish Government (DPI2009-10518 MICINN) and the Madrid Community (CCG07-UC3M/AMB-3412 and CCG08-UC3M/AMB-4227).

\section{Appendix A. Nomenclature}

$\begin{array}{ll}D & \text { bed diameter } \\ D_{O} & \text { object diameter } \\ f & \text { frequency } \\ f_{b} & \text { characteristic frequency of the bed } \\ H & \text { vessel height } \\ h_{b} & \text { bed height } \\ L_{O} & \text { object length }\end{array}$

$n \quad$ rotating angular velocity

$N_{a p} \quad$ number of appearances of the object at the bed surface

$P \quad$ pressure

$P_{S C} \quad$ stagnation cycle probability

$U \quad$ air velocity

$U_{m f} \quad$ minimum fluidization velocity

$\Delta P_{\text {dist }}$ pressure drop across the distributor

$\varepsilon_{\text {packed }} \quad$ void fraction of the packed bed

$\rho_{b} \quad$ packed bed density

$\rho_{o} \quad$ object density

\section{References}

[1] M. Stein, Y.L. Ding, J.P.K. Seville, D.J. Parker, Solids motion in bubbling gas fluidised beds, Chemical Engineering Science 55 (2000) 5291-5300.

[2] T. Grassler, K.-E. Wirth, X-ray computer tomography-potential and limitation for the measurement of local solids distribution in circulating fluidized beds, Chemical Engineering Journal 77 (1-2) (2000) 65-72.

[3] B. Du, F. Wei, W. Warsito, Gas and solids mixing in a turbulent fluidized bed, AIChE Journal 48 (9) (2002) 1896-1909.

[4] L. Shen, F. Johnsson, B. Leckner, Digital image analysis of hydrodynamics twodimensional bubbling fluidized beds, Chemical Engineering Science 59 (2004) 2607-2617.

[5] G.A. Bokkers, M. van Sint Annaland, J.A.M. Kuipers, Mixing and segregation in a bidisperse gas-solid fluidised bed: a numerical and experimental study, Powder Technology 140 (3) (2004) 176-186.

[6] C.H. Ibsen, T. Solberg, B.H. Hjertager, F. Johnsson, Laser Doppler anemometry measurements in a circulating fluidized bed of metal particles, Experimental Thermal and Fluid Science 26 (6-7) (2002) 851-859.

[7] D. Kunii, O. Levenspiel, Fluidization Engineering, 2nd ed., ButterworthHeinemann, Boston, 1991.

[8] G.M. Rios, K. Dang Tran, H. Masson, Free object motion in a gas fluidized bed, Chemical Engineering Communications 47 (1986) 247-272.

[9] A.W. Nienow, P.N. Rowe, T. Chiba, Mixing and segregation of a small portion of large particles in gas fluidized beds of considerably smaller ones, AIChE Symposium Series 74 (1978) 45-53.

[10] K.S. Lim, P.K. Agarwal, Circulatory motion of a large and lighter sphere in a bubbling fluidized bed of smaller and heavier particles, Chemical Engineering Science 49 (3) (1994) 421-424.

[11] A.C. Rees, J.F. Davidson, A.N. Hayhurst, The rise of a buoyant sphere in a gasfluidized bed, Chemical Engineering Science 60 (2005) 1143-1153.

[12] T.H. Nguyen, J.R. Grace, Forces on objects immersed in fluidized beds, Powder Technology 19 (1978) 255-264.

[13] H. Tanimoto, S. Chiba, T. Chiba, H. Kobayashi, Jetsam descent induced by a single bubble passage in three-dimensional gas-fluidized beds, Journal of Chemical Engineering of Japan 14 (4) (1981) 273-276.

[14] A.C. Hoffmann, L.P. Janssen, J. Prins, Particle segregation in fluidized binary mixtures, Chemical Engineering Science 48 (9) (1993) 1583-1592.

[15] J. Sanderson, M. Rhodes, Hydrodynamic similarity of solids motion and mixing in bubbling fluidized beds, AIChE Journal 49 (9) (2003) 2317-2327.

[16] S.A. Keillor, M.A. Bergougnou, A study of the action of floating bubble breakers in fluidized beds by interactive computer graphics, Fluidization Technology 2 (1975) 95-109.

[17] N.A. Moussa, A.A. Fowle, M.M. Delichatsios, R.N. Caron, R. Wilson. Advanced design for pulsed atmospheric fluidized bed combustion. Final Report to DOE/METC, Morgantown Energy Technology Center, 1982.

[18] J.R. van Ommen, J. Nijenhuis, C.M. van den Bleek, M.O. Coppens, Four ways to introduce structure in fluidized bed reactors, Industrial \& Engineering Chemistry Research 46 (2007) 4236-4244.

[19] K. Noda, Y. Mawatari, S. Uchida, Flow patterns of fine particles in a vibrated fluidized bed under atmospheric or reduced pressure, Powder Technology 99 (1998) 11-14.

[20] Y. Mawatari, Y. Tatemoto, K. Noda, Prediction of minimum fluidization velocity for vibrated fluidized beds, Powder Technology 131 (2003) 66-70.

[21] F. Ouyang, O. Levenspiel, Spiral distributor for fluidized beds, Industrial \& Engineering Chemistry Process Design and Development 25 (1986) 504-507.

[22] B. Sreenivasan, V.R. Ragahavan, Hydrodynamics of a swirling fluidized bed, Chemical Engineering Science 41 (2002) 99-106.

[23] C.S. Chyang, Y.C. Lin, A study in the swirling fluidizing pattern, Journal of Chemical Engineering of Japan 35 (2002) 503-512.

[24] C. Sobrino, J.A. Almendros-Ibáñez, D. Santana, M. de Vega, Fluidization of Group B particles with a rotating distributor, Powder Technology 181 (2008) 273-280.

[25] C. Sobrino, A. Acosta-Iborra, D. Santana, M. de Vega, Bubble characteristics in a bubbling fluidized bed with a rotating distributor, International Journal of Multiphase Flow 35 (10) (2009) 970-976.

[26] S.B.R. Karri, J. Werther, Gas distributor and plenum design in fluidized beds, in: W.C. Yang (Ed.), Handbook of Fluidization and Fluid-particle Systems, Marcel Dekker Inc., New York, 2003, pp. 164-179.

[27] D. Geldart, J. Baeyens, The design of distributors for gas-fluidized beds, Powder Technology 45 (1985) 67-78.

[28] A.C. Rees, J.F. Davidson, J.S. Dennis, P.S. Fennell, L.F. Gladden, A.N. Hayhurst, M.D. Mantle, C.R. Müller, A.J. Sederman, The nature of the flow just above the 
perforated plate distributor of a gas-fluidised bed, as imaged using magnetic resonance, Chemical Engineering Science 61 (2006) 6002-6015.

[29] H. Bergh, H. Tijdeman. Theoretical and experimental results for the dynamic response of pressure measuring systems. Report NLR-TR F.238, National Aeroand Astronautical Research Institute, Amsterdam, the Netherlands, 1965.

[30] J.R. van Ommen, J.C. Schouten, L.M. vander Stappen, C.M. van den Bleek, Response characteristics of probe transducer systems for pressure measurements in gas solid fluidized beds how to prevent pitfalls in dynamic pressure measurements, Powder Technology 106 (1999) 199-218.

[31] P.D. Welch, The use of a fast Fourier transform for the estimation of power spectra, IEEE Transaction Audio and Electroacoustics AU-15 (1967) 70-73.

[32] F. Johnsson, R.C. Zijerveld, J.C. Schouten, C.M. van den Bleek, B. Leckner, Characterization of fluidization regimes by time-series analysis of pressure fluctuations, International Journal of Multiphase Flow 26 (2000) 663-715.
[33] J. Werther, O. Molerus, The local structure of gas fluidized beds-II. The spatial distribution of bubbles, International Journal of Multiphase Flow 1 (1973) 123-138.

[34] R.C. Darton, R.D. LaNauze, J.F. Davidson, D. Harrison, Bubble growth due to coalescence in fluidized beds, Transactions of the Institute of Chemical Engineering 55 (1977) 274-280.

[35] D. Pallarès, F. Johnsson, A novel technique for particle tracking in cold 2 dimensional fluidized beds-simulating fuel dispersion, Chemical Engineering Science 61 (2006) 2710-2720.

[36] D. Geldart, Types of gas fluidization, Powder Technology 7 (1973) 285-292.

[37] J. Otshitani, O. Keiko, I. Makoto, T. Zennosuke, Effect of particle fluidization intensity on floating and sinking of objects in a gas-solid fluidized bed, Advanced Powder Technology 15 (2) (2004) 201-213. 